\title{
Pharmacopuncture for Acute peripheral facial paralysis: A systematic review and meta-analysis of randomized controlled trials
}

\author{
Min-Sun $\mathrm{Choi}^{1}$, Dong Il Kim ${ }^{1}$, Su Hee Jang ${ }^{2}$ \\ ${ }^{1}$ Department of Obstetrics \& Gynecology, College of Korean Medicine, Dongguk University, \\ Ilsan, Republic of Korea \\ ${ }^{2}$ Department of Acupuncture \& Moxibustion, Nasaret Korean Medical Hospital, Incheon, Republic of Korea
}

\begin{abstract}
Objectives: This study aims to evaluate the efficacy of pharmacopuncture in the treatment of acute peripheral facial paralysis (APFP) through a meta-analysis of randomized controlled trials to assess the clinical evidence for pharmacopuncture as a treatment for PFP.

Methods: Literature was searched using databases. The search terms used were "pharmacopuncture," "sweet BV," "apipuncture," "apitoxin," "injection acupuncture," and "herbal acupuncture" in English and "bongchim," "bongyakchim," and "yakchim" in Korean. The RevMan 5.2 program was used for meta-analysis.

Results: In all, 1902 studies were screened and the full texts of 219 articles were reviewed. Of these, nine studies were included in the systematic review and nine studies were included in the meta-analysis. Treatments utilizing bee venom, sweet bee venom (SBV), Soyeom, Calculus Bovis $\cdot$ Fel Ursi $\cdot$ Moschus (BUM), Hominis Placenta, and Suseunghwagang (SSHG) were observed in nine RCTs. In the forest plot, the diamond favors the PA treatment against the control treatment. (95\% CI: $0.34[0.08,0.60], \mathrm{Z}=2.57, \mathrm{P}=0.01)$, which Our meta-analysis in RevMan 5.2 confirmed the significant efficacy of PA in treating APFP.

Conclusion: Our systematic review and meta-analysis suggest that treatments using Soyeom, bee venom, and BUM are effective in APFP and they reduce the duration of postauricular pain occurring at the acute stage of APFP. However, due to small effect size, further investigation is needed.
\end{abstract}

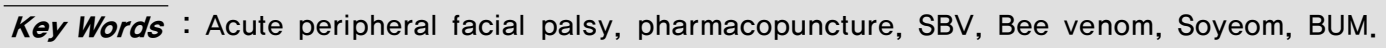

\section{Introduction}

Acute peripheral facial paralysis (APFP) is a relatively common disease of the facial area. Its incidence varies from 20 to 30 cases per 100,000 people with the incidence increasing with age $[1,2]$.

In addition, it is the most common cause of unilateral facial paralysis, accounting for $60 \%$ to $75 \%$ of all cases of damage to the seventh cranial nerve [2]. APFP is characterized by unilateral facial weakness and the inability to control movement in the facial muscles; other symptoms include loss of taste, hyperacusis, decreased salivation, and tear secretion. Acute facial pain radiating from the ear may precede the onset of other symptoms [3, 4]. Several studies provide increasing evidence of the beneficial effect of acupuncture and moxibustion as an adjunctive treatment for APFP [5-7]. In

\footnotetext{
- Received : 16 February 2015

- Revised : 16 June 2015

- Accepted : 22 June 2015

- Correspondence to: Su Hee Jang

Department of Acupuncture \& Moxibustion, Nasaret Korean Medical Hospital, Incheon, Republic of Korea Inchoen Namdonggu Gansuk 2dong 207-1

Tel : +82-32-435-5111, Fax : +82-32-435-5115, E-mail : jangsuhe@gmail.com
} 
conjunction with acupuncture and herbal medicine, pharmacopuncture (PA) is being used for the treatment of APFP. PA has been utilized in various types of disease as a complimentary method to conventional acupuncture treatment; however, no evidence shows its effectiveness for APFP. Therefore, the aim of this systematic review and meta-analysis is to investigate the effect of PA as an adjunctive treatment given in conjunction with the conventional Korean medical treatment for APFP.

\section{Methods}

\section{Data Sources and Searches}

The literature search was performed separately on April 3, 2014 by two investigators, who are Korean medical doctors. Only results published in English or Korean were considered. Data were recorded and assessed using Microsoft Excel 2007. The following electronic databases were searched from inception through April 2, 2014: Pubmed, Elsevier-EMBASE, Cochrane Central Register of Controlled Tirals (CENTRAL) (The Cochrane Library), SCOPUS, Cumulative Index to Nursing and Allied Health Literature (CINAHL), Citation Information by National Institute of Informatics (CiNii), Korean-studies Information Service System (KISS), DBPIA, KoreaMed. We used the following terms in English: pharmacopuncture, sweet bee venom, apipuncture, apitoxin, injection acupuncture, aquapuncture, herbal acupuncture. In Korean: bongchim, bongyakchim, yakchim, and suchim.

\section{Study Selection}

The study selection was performed independently by two investigators. Duplicate studies were excluded from the Excel database of articles by comparing the title, author, and year published. Inappropriate studies were excluded by examining the title and the abstract.

\section{1) Types of studies}

Studies mentioning randomized controlled trials (RCTs) of PA for APFP were considered. Studies that did not mention random allocation method, non-randomized controlled trials (nRCTs), case studies, and experimental studies were excluded.

\section{2) Types of participants}

The patients selected were diagnosed with APFP, and their initial treatment began within ten days of onset.

\section{3) Types of intervention}

The interventions selected involved the PA of any ingredient to any injection point with no limit on the total dosage per injection per treatment session.

\section{4) Control group}

No restrictions were placed on the type of control. Normal saline, conventional acupuncture treatments or various PA ingredients were included.

\section{5) Types of outcome measurements}

The outcome measure was set to the Yanagihara -system to assess APFP at the end of the fourth week as the primary outcome. The second and third week scores were also compared by grouping the trials according to the similar control groups. The VAS score for postauricular pain was examined at the end of the treatment session.

\section{Data Extraction}

Data extraction was performed independently by two unblinded investigators, and discrepancies were resolved through discussion. Data from the articles were validated and extracted using a predefined data extraction form. The study characteristics examined were sample size, age range, intervention, treatment duration, outcome, and result. The outcomes included symptoms and adverse events. 


\section{Risk of Bias Assessment}

Risk of bias (ROB) assessment was independently performed by the two reviewers, using the Cochrane Handbook for Systematic Reviews of Interventions [8]. In addition, the Jadad score [9] was used as a complimentary assessment. The reviewers had no disagreements regarding the risk of bias.

\section{Data Synthesis: Estimation Statistics}

To summarize the effects of PA on outcomes, Review Manager (RevMan) (Version 5.2., Nordic Cochrane Centre, Cochrane Collaboration, Copenhagen) was used. The effect size was calculated as weighted mean differences (WMDs) and a 95\% confidence interval (95\% CI). Random-effect models were employed to consider the possible clinical heterogeneity among the included studies using the chi-square test, the tau ${ }^{2}$ test, and Higgins $I^{2}$ statistics [8]).

\section{Results}

\section{Study Selection}

The titles and/or abstracts of 1902 studies were screened, and the full texts of 219 studies were reviewed (Figure 1). Studies declared ineligible based on their titles and/or abstracts were excluded.
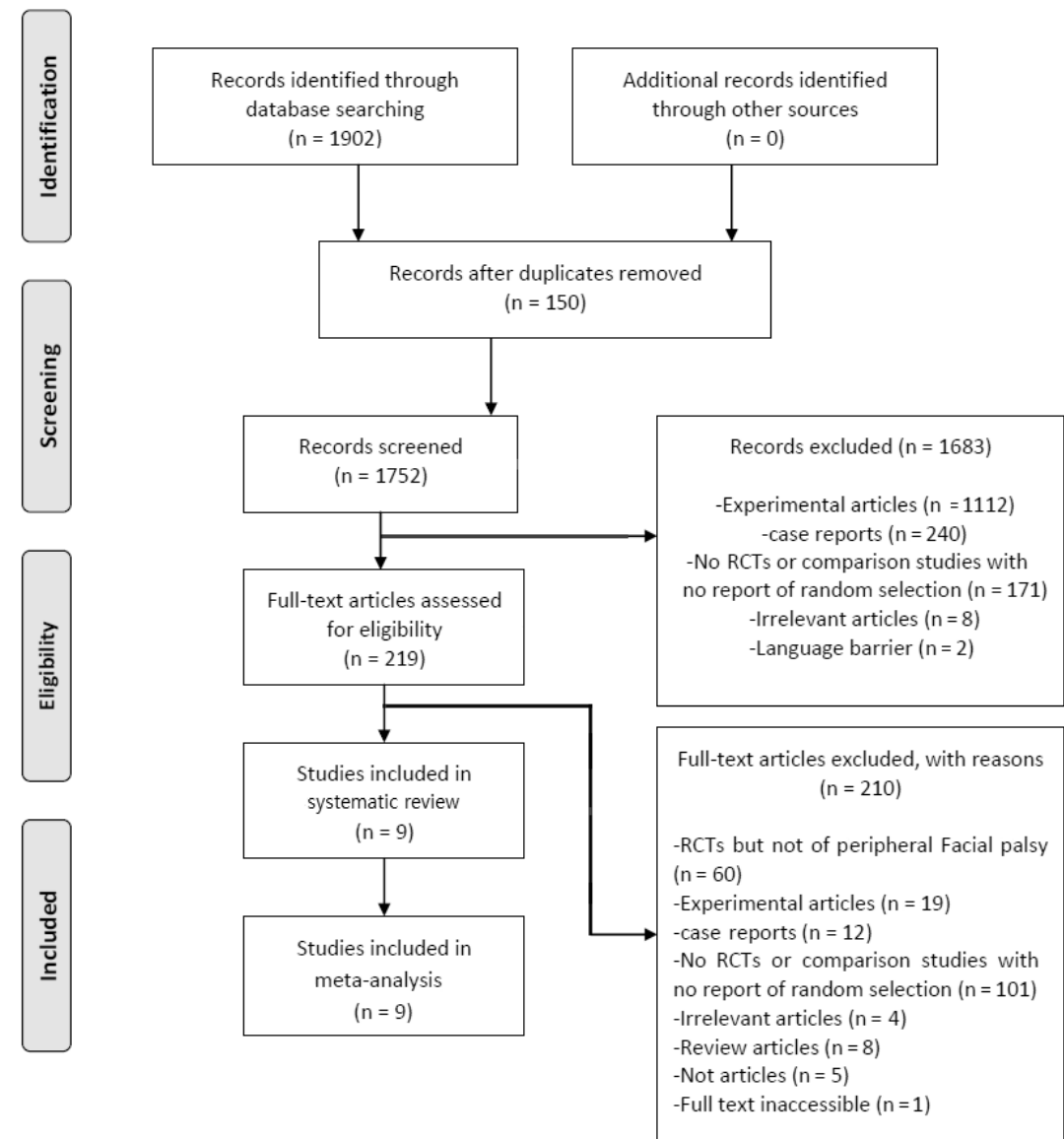

Fig. 1. PRISMA flowchart of study selection. RCTs: Randomized controlled trials. 
Ineligible studies that were not filtered out during the title and/or abstract screening stage were found and removed after reviewing the full text. Of the 219 articles reviewed in full text, nine studies were identified for inclusion in the systematic review and meta-analysis [10-18]. Of the nine studies, four articles focusing on postauricular pain were included [10-12, 18].

\section{Study description}

\section{1) Characteristics of included studies}

In all, nine studies were included for systematic review and meta-analysis. The summary of the included RCTs are presented in Table 1 and 2. All nine RCTs originated from the Republic of Korea, and all the trials adopted an open-label RCT, two-armed parallel group design. In all, 200 patients were included in these PA treatment trials. Of those, 76 patients were included in studies on the effect of PA on APFP with postauricular pain. None of the RCTs had a treatment group receiving PA as a single intervention. Instead, PA in all the trials was combined with conventional Korean medical treatments, including acupuncture, herbal medicine, and other physical therapies (e.g., silver spike point therapy (SSP), hot pack, interferential current therapy (ICT), cupping therapy, and facial massage and exercise). Overall, six different PA ingredients were studied. Soyeom, bee venom, Calculus Bovis $\cdot \mathrm{Fel}$ Ursi $\cdot$ Moschus (BUM), sweet bee venom (SBV), Suseunghwagang (SSHG), and Hominis Placenta (HP) were used in the treatment group. In studies on APFP with postauricular pain, Soyeom, bee venom, and BUM were used in the treatment group. Different types of controls were used in the studies. Lee CW (2004) used a placebo controlled with normal saline. Four RCTs compared PA with conventional Korean medical treatment $[10,11,14$, 18]. Three RCTs compared PA treatments with different PA treatments [12, 15, 17]. Kim JH (2013) compared PA with needle-embedding therapy, which is a unique method of acupuncture in which absorbable needles are left in the subcutaneous tissue in the affected region.

\section{2) Characteristics of pharmacopuncture intervention} Soyeom, Bee venom, BUM, SBV, SSHG, and Hominis Placenta have been studied (Table 3). Of nine studies, four trials used herbal formula compounds $[10,12,15,18]$. Six trials used single-herb PA [11, 13-17]. Lee $\mathrm{CH}$ et al. (2011) compared single-herb with mixed-herb PA, and Choi YJ et al. (2012) compared mixed-herb PA with formulated ingredients. Of the 200 patients with APFP, 47 were treated with Soyeom, 83 were treated with bee venom, 14 were treated with BUM, 15 were treated with SSHG, and 41 were treated with Hominis Placenta. Of the 76 patients with postauricular pain, 47 were treated with Soyeom, 15 were treated with bee venom, and 14 were treated with BUM. All trials used 5 10 acupuncture points in the facial area; for one group, Lee $\mathrm{CH}$ et al. (2011) included two acupuncture points not located on the face. Four trials studying postauricular pain chose TE17 as the injection point [13, 14, 22, 25]. Of these, Kim MS et al. (2004) was the only trial to report the assessment of skin sensitivity before the test for any allergic reaction and was the only trial to report any adverse events.

\section{3) Outcome Measures}

All the trials examined in this study used the Yanagihara-system as the primary outcome (the change of Yanagihara system). In addition, four postauricular pain trials used VAS scores [13, 14, 25]. Kim JH et al. (2013) also used HBS. Choi BC et al. (2009) did not report on the treatment duration and only reported the end scores. Four trials reported weekly scores and improvement rates for four weeks of treatment [13-15, 17]. Lee CW et al. (2004) reported weekly scores for five weeks of treatment 
(104) Journal of Korean Medicine 2015;36(2)

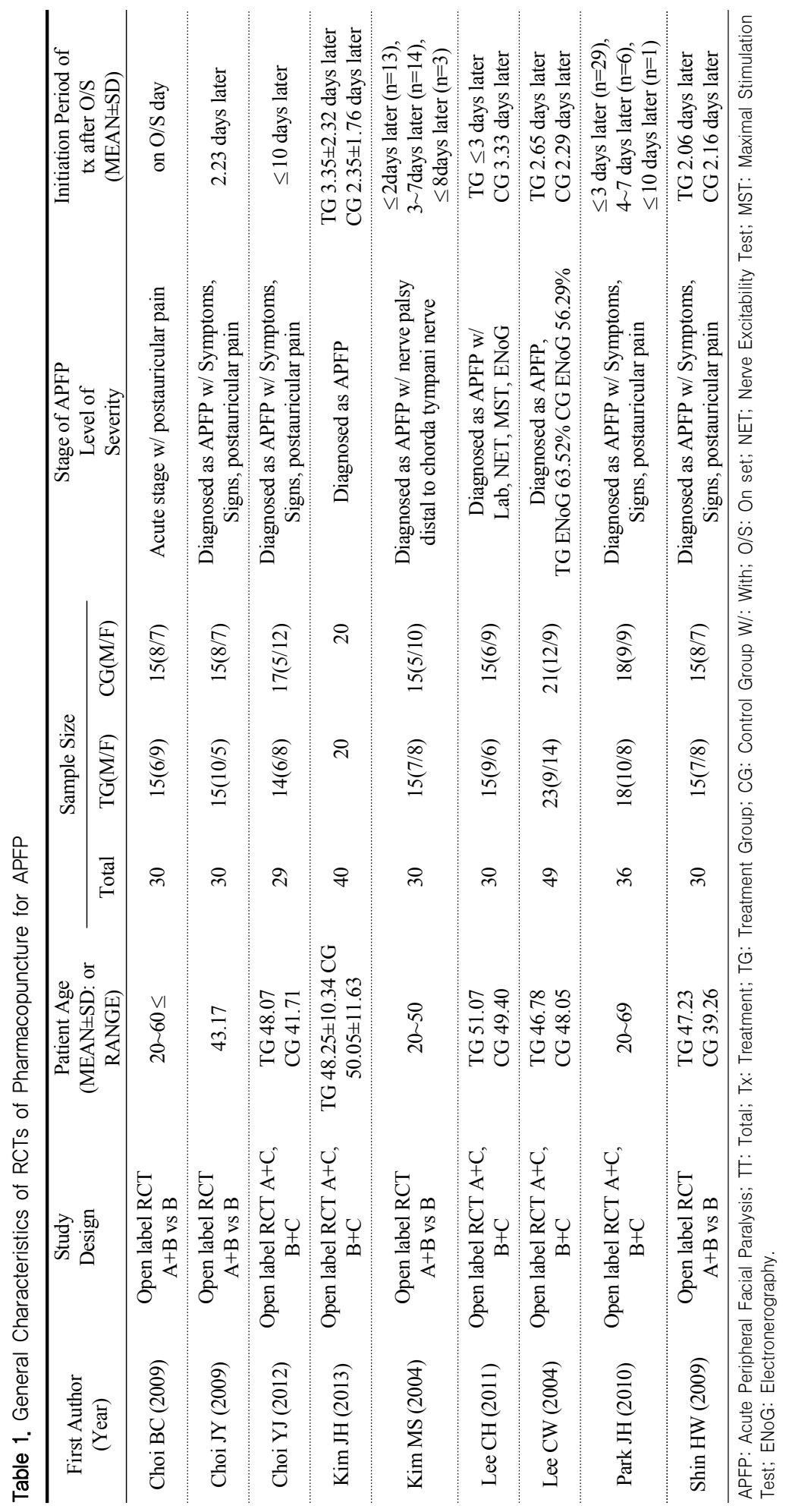




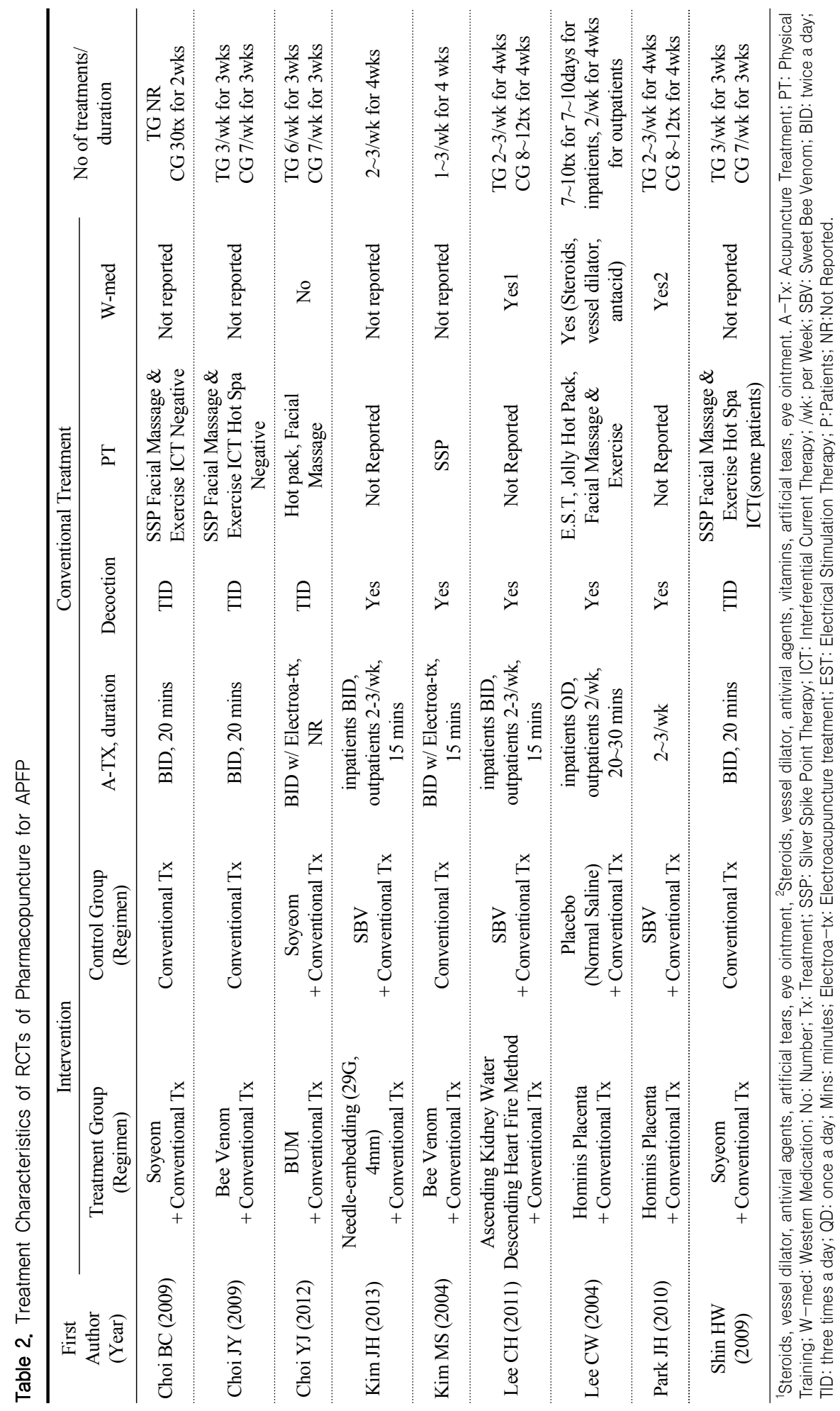


(106) Journal of Korean Medicine 2015;36(2)

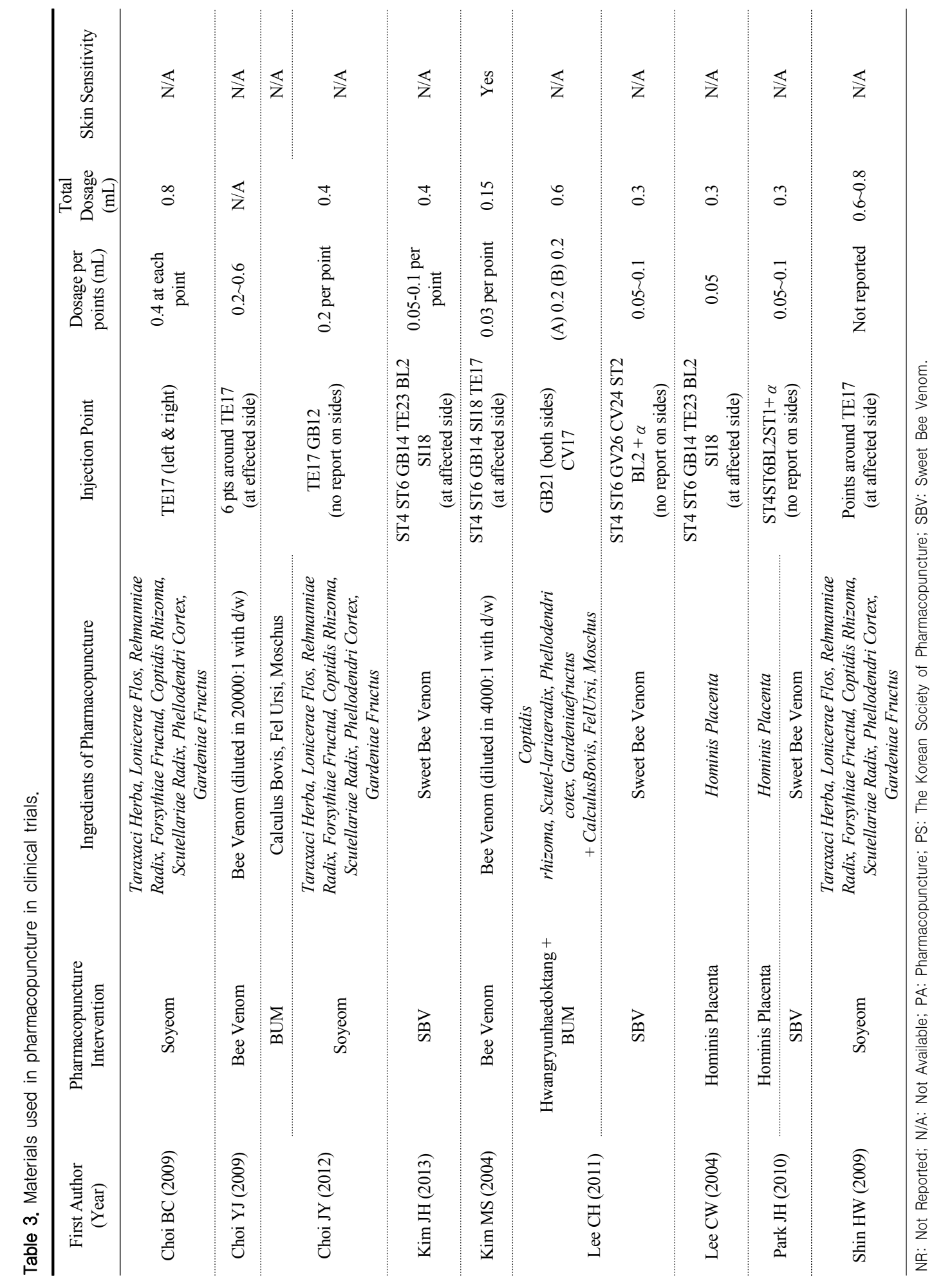


but did not report improvement rates. Three trials reported only the initial and end scores and the improvement rates with VAS scores for the second, fourth, and sixth days of treatment $[11,12,18]$. Choi $\mathrm{BC}$ et al. (2009) reported scores from the seventh, fifteenth, and last days of treatment and the improvement rates with VAS scores for the third, fifth, and seventh days of treatment. The quality of life was not assessed in any of the trials examined. Kim MS et al. (2004) was the only trial to report adverse events following the injection.

\section{Risk of Bias Assessment}

The Cochrane Handbook was used to assess the risk of bias to review the studies for methodological quality and the risk of bias [8]. Four trials used random sequence generation $[13,15,17,18]$. None of the trials showed allocation concealment. Six trials had a high risk for performance bias. Only three trials had two parallel groups that used the same intervention techniques [12, 16, 17]. None of the trials showed detection bias, reporting bias, or any other bias. Kim MS et al. (2004) was at high risk for attrition bias because of drop-outs in the experimental group due to adverse events (Figures 2 and 3). According to the Jadad scoring system, all the trials were randomized in various ways and therefore received 1 point; however, only four trials used appropriate methods $[5,13,17,18]$. Three trials used randomization codes $[5,13,17]$. Park JH et al.

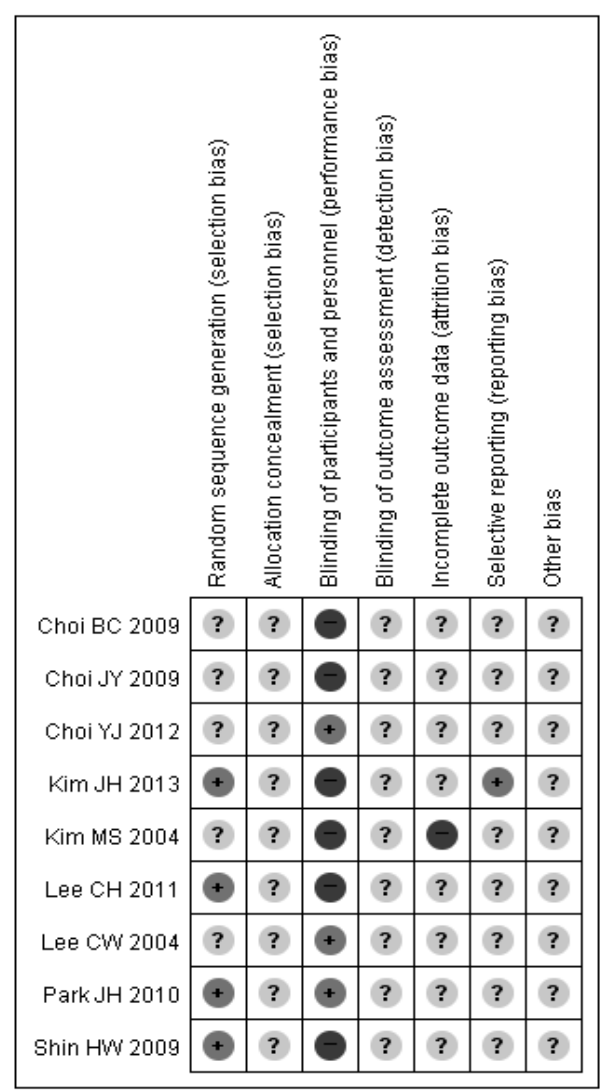

Fig. 2. The risk of bias of included studies. +: Low risk of bias; -: High risk of bias; ?: Unclear.

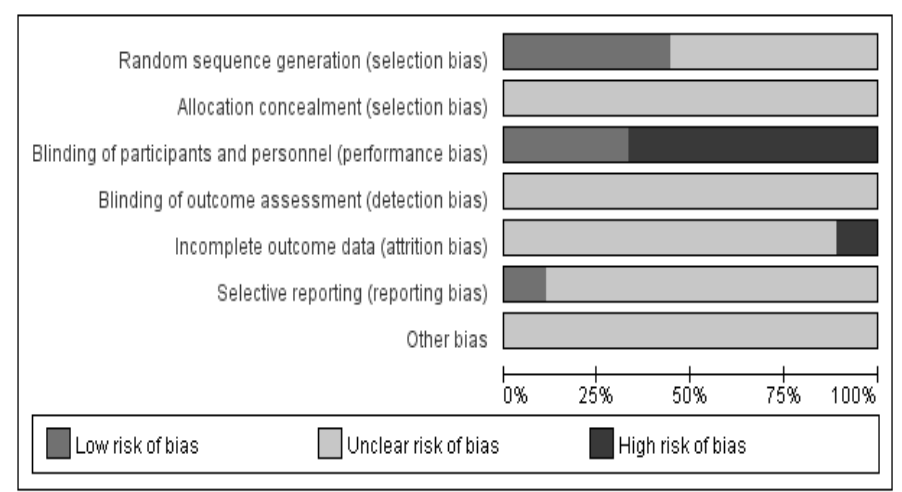

Fig. 3. The summary of risk of bias 
(2010) tossed a coin to randomize. None of the trials used blinding, and three trials included drop-outs or withdrawals $[13,14,15]$. Thus, none of the trials obtained a score of 5, which indicates a high-quality RCT. Only two studies received a total score of 3 $[13,15]$. Three studies received a score of $2[14,17$, 18]. The rest of the trials received a score of 1 . None of the trials received a score of negative 1.

\section{Outcome Results}

The data extracted from the included studies were classified according to the weekly assessment results, with the fourth week used as the primary outcome in the analysis of efficacy (Figure 4). Only three studies that had conventional Korean medical treatment or normal saline as control were used for the primary outcome during the fourth week of treatment. The meta-analysis showed no heterogeneity among the three studies. The result of primary outcome was significant with Relative Ratio $(\mathrm{RR})=0.35\left(\chi^{2}=\right.$ 2.79, $\mathrm{P}=0.25, I^{2}=28 \%$ ). The diamond in the forest plot was on the right side of the vertical line, but it did intersect with the line. We found 95\% CI for -0.09 to $0.79(\mathrm{Z}=1.54, \mathrm{P}=0.12)$, which meant that the PA treatment was better than the control treatment. When comparing the primary outcome (fourth week of treatment) with the secondary outcome (second and third weeks of treatment), the data shows that the diamond intersects with the line at the second week of treatment, thus indicating that the result does not favor PA. However, at the third week of treatment, the diamond does not intersect with the vertical line. Kim JH et al. (2013) used the embedded-needling method of acupuncture and showed that this method and PA are both effective but with no significant differences. No heterogeneity

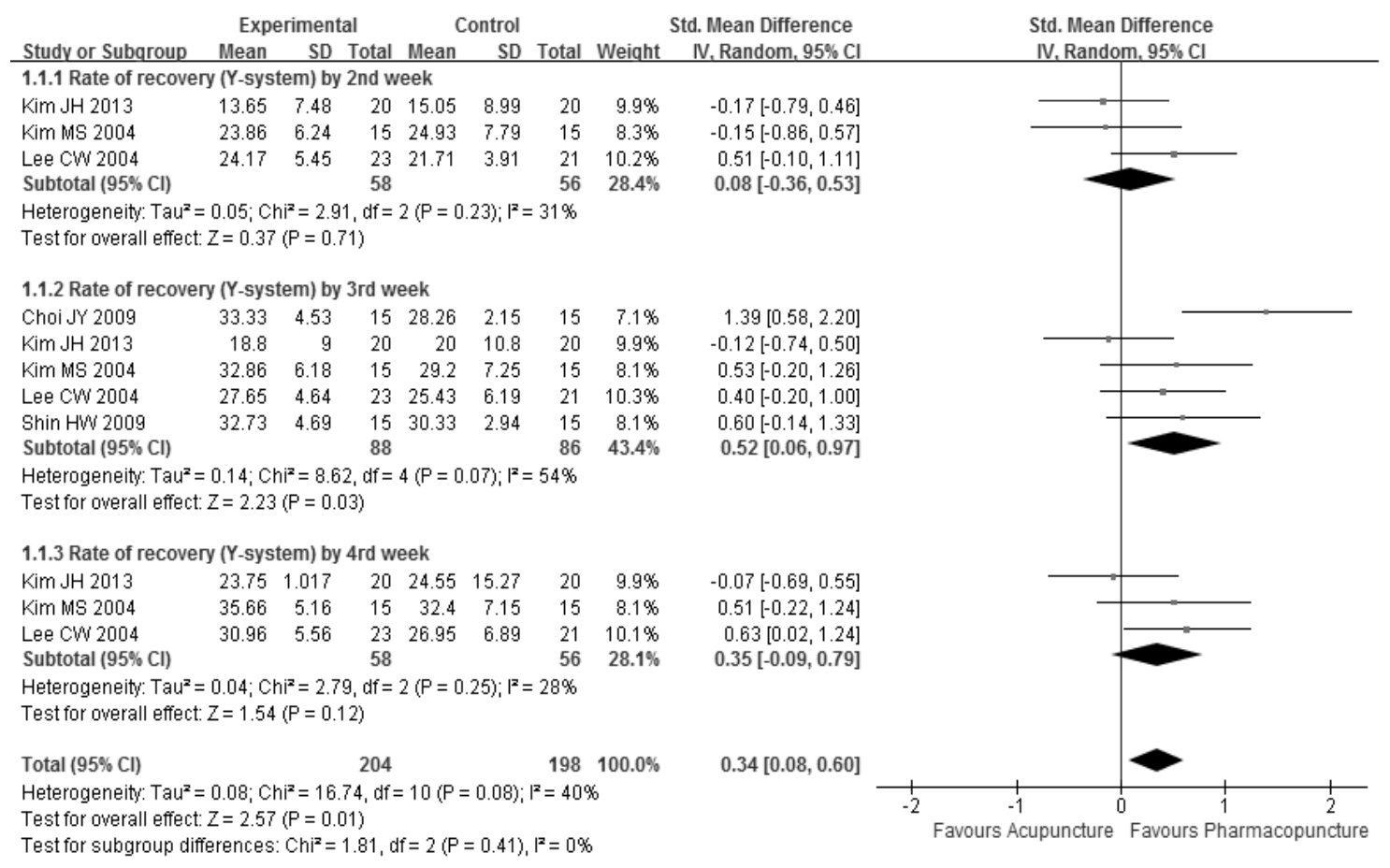

Fig. 4. The forest plot of Yanagihara-system score by weekly treatment. All controls were conventional Korean medical treatments except for Kim JH (2013), which used the embed-needling technique. 
and no significance was seen in the second week, with $\mathrm{RR}=0.08\left(\chi^{2}=2.91, \mathrm{P}=0.23, I^{2}=31 \%\right)$. At the third week, the heterogeneity among the two included studies was significant $\left(\chi^{2}=8.62, \mathrm{P}=0.07\right.$, $I^{2}=54 \%$ ). Overall, $\mathrm{RR}=0.34$ with a $95 \% \mathrm{CI}$ of 0.08 to 0.60 with heterogeneity present $\left(\chi^{2}=16.74, \mathrm{P}=0.08\right.$, $\left.I^{2}=40 \%\right)$ and an overall effect of $\mathrm{Z}=2.57(\mathrm{P}=0.01)$. The test for subgroup differences had no heterogeneity ( $\chi^{2}=1.81, \mathrm{P}=0.41, I^{2}=0 \%$ ), thus favoring the $\mathrm{PA}$ method.

\section{1) Outcome results in the RCTs on VAS for postauricular pain}

The scores related to the use of VAS for postauricular pain were extracted from the included studies and analyzed to determine its efficacy in controlling pain and shortening the duration of pain (Figure 6). Four pieces of data from the sixth day of treatment were used. The meta-analysis showed that the heterogeneity among the four studies included was significant ( $\chi^{2}=6.51, \mathrm{df}=3, \mathrm{P}=0.09, I^{2}=54 \%$ ). The diamond was on the left side of the vertical line in the forest plot, and it did not intersect with the line. We found that $\mathrm{RR}=-0.95$ with a $95 \% \mathrm{CI}$ of -0.95 to $-0.38(\mathrm{Z}=3.28, \mathrm{P}=0.001)$, which meant that PA shortened the duration of and reduced postauricular pain by $15 \%$.

\section{Discussion}

Recently, PA was introduced as an improved method to compliment conventional acupuncture treatment. When used as a complimentary treatment to acupuncture, PA is much more effective [20]. PA, which involves the integration of acupuncture and herbal medicine, has been one of the most widely used acupuncture treatment methods in Korean medicine since Nam Sang Chun began its clinical utilization in 1967. However, its historical origin dates back to BC 168 in China. At the beginning of the $20^{\text {th }}$ century, PA began to be used in Korea [21]. RCTs on PA were screened; in all, nine RCTs were found that focused on APFP. Although the search for RCTs was performed using international search engines, all the RCTs included in this study originated from the Republic of Korea. Our review of the RCT studies compared PA used in conjunction with conventional Korean medical treatments with conventional Korean medical treatments alone or with other PA treatments. Choi BC (2009) lacked standard deviation information for the weekly scores; this author tried to reach the author of the study by phone and email but received no reply. Therefore, only the VAS score was used. Trial observations were performed, with a total of 200 patients observed in the PA groups (Soyeom: 47 patients; SBV: 53; bee venom: 30; SSHG: 15 ; HP: 41; BUM: 14). The results suggest that Soyeom controls pain during the initial treatment stage; one study on Soyeom showed that it was significantly effective for APFP [12]. In addition, Soyeom relieved and shortened the duration of postauricular

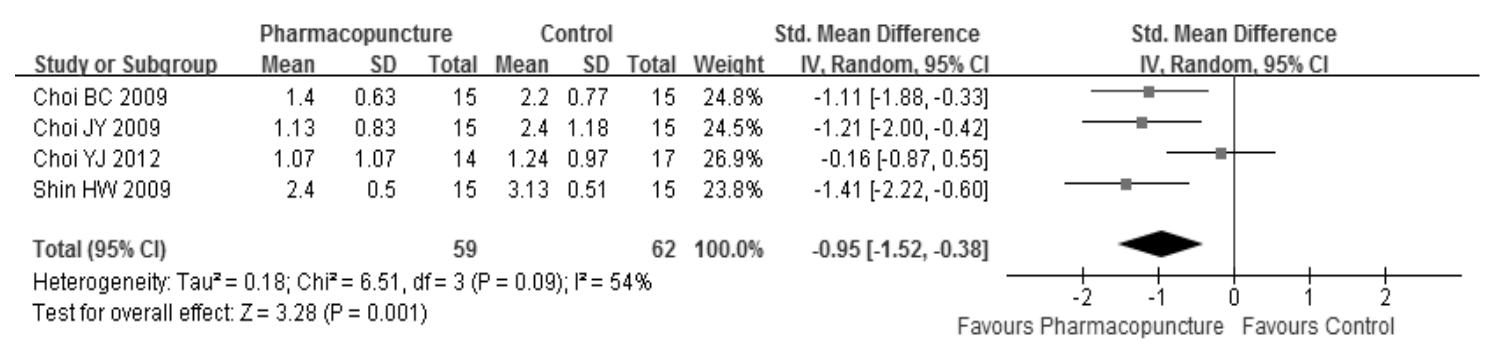

Fig. 5. The forest plot of VAS score on postauricular pain based on the sixth day of the treatment 
pain $[12,18]$. In Shin HW study on Soyeom, the duration of postauricular pain lasted for $8.53 \pm 1.24$ days compared to $7.06 \pm 1.44$ days for control group. There was only about 1.5 day difference between the two groups thus Soyeom may be the most effective at the very acute stage of pain. Two studies on bee venom and SBV showed effectiveness at two weeks and thereafter; thus, they were effective in the acute stage $[13,15]$. In Choi JY study, Bee venom did not show significant results on reducing the degree of pain, but significant difference was seen on duration on pain. Bee venom group had the lasting postauricular pain for $5.86 \pm 1.35$ days compared to $8.26 \pm 1.9$ days for the control group. BUM also shortened the duration of postauricular pain; however, no significant difference was seen between the control groups related to the improvement of APFP. Hominis Placenta showed results in the second, third, and fourth weeks but with no significance. SSHG also showed results with no significance. Yanagihara system grades the degree of APFP under ten category; at rest, wrinkle forehead, blink, closure of eye lightly, closure of eye tightly, closure of eye involved side only, wrinkle nose, whistle, grin, and depress lower lip with each category scored in the scale of 0 to 4 . Unfortunately, none of the studies mentioned detailed description of improvement on APFP of those ten categories. To rank the most effective PA for APFP, more studies should be performed. The current evidence lacks enough information regarding the termination of the treatment and the number of treatment sessions needed for the best results. Consequently, the real efficacy of PA for APFP remains unclear because the quality of some of the articles included in the systematic review and meta-analysis were poor. We performed the heterogeneity test using the $\chi^{2}$ test ( $\chi^{2}=1.81, \quad \mathrm{P}=0.41, \quad I^{2}=0 \%$ ) We found that the included studies did not cause heterogeneity. All the trials included here were randomized in various ways. Kim JH et al. (2013) and Lee $\mathrm{CH}$ et al.
(2011) used randomization codes and Park JH et al. (2010) tossed a coin. None reported subject or evaluator blinding. Thus, no RCTs had a Jadad score of 4 or 5 . Nonetheless, all the RCTs had a high risk of bias. In addition, the RCTs except Kim MS et al. (2004) were missing information on adverse effects; therefore, the safety of PA is also unclear. The RCTs showed no publication bias.

\section{Conclusion}

Nine randomized clinical trials were included in this systematic review and meta-analysis, which focused on Soyeom, bee venom, SBV, BUM, HP, and SSHG. The evidence from all the RCTs examined in this study was positive except for those from Kim JH et al. (2013). Although the results vary, our findings suggest the effectiveness of Soyeom, bee venom, and SBV for APFP and of Soyeom and BUM for postauricular pain at acute stage. However, further research is required to assess more accurate results and PA's mechanism of action in the treatment of APFP.

\section{References}

1. Pereira C, Santos E, Monteiro J, Morais AA, Santos CT. Peripheral Facial Palsy: Anatomy And Physiology. An Update. The Internet Journal of Neurosurgery. 2004;2(2).

2. Steiner I, Mattan Y. Bell's palsy and herpes viruses: to (acylo)vir or not to (acylo)vir? J Neurol Sci 1999;170:19-23.

3. Colledge NR, Walker BR, Ralston S, Davidson S. Davidson's principles and practice of medicine. 21st ed. Edinburgh:Churchill Livingstone/ Elsevier. 2010.

4. Fauci AS, Harrison TR, et al. Harrison's principles of internal medicine. 17th ed. New York[etc.]:McGraw-Hill Medical. 2008.

5. Lee CK. Acupuncture Trials for Peripheral 
Facial Palsy in Republic of Korea: Systematic Review The Acupuncture. 2013;30(3):51-59.

6. Liang F, Li Y, Yu S, Li C, Hu L, Zhou D, et al. A multicentral randomized control study on clinical acupuncture treatment of Bell's palsy. J Tradit Chin Med. 2006;26:3-7.

7. Qu Y. Clinical observation on acupuncture by stages combined with exercise therapy for treatment of Bell palsy at acute stage. Zhongguo Zhen Jiu. 2005;25:545-547.

8. Higgins JPT, Altman DG. Chapter 8: Assessing risk of bias in included studies. In: Higgins JPT, Green S, (editors). Cochrane Handbook for Systematic Reviews of Interventions. Chichester: The Cochrane Collaboration, John Wiley \& Sons. 2008:187-242.

9. Jada Ar, Moore RA, Carroll D, Jenkinson C, Reynolds DJ, Gavaghan DJ, et al. Assessing the quality of reports of randomized clinical trials: is blinding necessary? Conrol Clin Trials. 1996; 17(1):1-12.

10. Choi BC, Han KS, Ahn TW. Clinical comparison studies on 30 cases of Bell's palsy patient with postauricular pain by Anti-inflammatory pharmacopuncture \& Acupuncture and Herbal therapy. Dajeon University Korean Medicine Research Center Collection of dissertations. 2009;18(2):89-94.

11. Choi JY, Lee H, Kang JH, Kim YI, Kim JH, Lee SH, et al. Comparative Study of General Oriental Medical Treatment and Bee Venom Pharmacopuncture on Acute Peripheral Facial Paralysis Patient with Postauricular Pain. The J of Korean Acupuncture \& Moxibustion. 2009; 26(5)95-103.

12. Choi YJ, Kim JH, Yoon KJ, Yeo IH, Lee CK, Lee EY, et al. Comparative Study of BUM Pharmacopuncture and Soyeom Pharmacopunture on Peripheral Facial Paralysis with Postauricular Pain The Journal of Korean Acupuncture \& Moxibustion Medicine Society. 2012;29(5):31-37.
13. Kim JH, Jeong JY, Lee SW, Shin SY, Park JH, $\mathrm{Kim} \mathrm{CH}$, et al. Comparison of the Efficacy between Needle -embedding Therapy and Swee Bee Venom Pharmacopuncture Therapy on Peripheral Facial Paralysis. The Acupuncture. 2013;30(4):35-44.

14. Kim MS, Kim HJ, Park YJ, Kim EH, Lee EY. The clinical research of the efficacy of bee venom aqua-acupuncture on peripheral facial paralysis. The $\mathrm{J}$ of Korean Acupuncture \& Moxibustion. 2004;21(4);251-262.

15. Lee $\mathrm{CH}, \mathrm{Ku}$ JY, Park JA, Lee YH. Comparison of the Efficacy between Method of Regulating Ascending Kidney Water and Descending Heart Fire and Sweet Bee Venom Pharmacopuncture on Peripheral Facial Paralysis. The J of Korean Acupuncture \& Moxibustion Society. 2011; 28(4):85-92.

16. Lee CW, Kim HG, Heo SW, Jung KK, Ahn $\mathrm{CB}$, Song $\mathrm{CH}$, et al. The Clinical Study about Honilirls Placenta Herbal Acupuncture on Bell's Palsy. J of Korean Pharmacopuncture Institute. 2004;8(3): 87-97.

17. Park JH, Jang SH, Lee $\mathrm{CH}, \mathrm{Ku}$ JY, Jeun DS, Ahn CB, et al. The Clinical Research of the Effectiveness of Pharmacopuncture Complex Therapy on Peripheral Facial Paralysis Hominis Placenta Pharmacopuncture Therapy and Sweet Bee Venom Therapy. The J of Korean Acupuncture \& Moxibustion Society. 2010;27(2):79-87.

18. Shin HW, Kang JH, Lee H. Efficacy of Soyeom Pharmacopuncture on Postauricular Pain Accompanied with Peripheral Facial Paralysis. The Journal of Korean Acupuncture \& Moxibustion Society. 2009;26(6):41-49.

19. Ahrens A, Skarada D, Wallace M, Cheung JY, Neely JG. Rapid simultaneous comparison system for subjective grading scales grading scales for facial paralysis. Am J Otol. 1999; (20):667-671. 
(112) Journal of Korean Medicine 2015;36(2)

20. Strudwick MW, Hinks RC, Choy STB. Point injection as an alternative acupuncture technique -an exploratory study of responses in healthy subjects. Acupuncture in Medicine. 2007;25(4): 166-174.

21. Kwan KL, Kim SC, Kim CH, No JD, Suh HS,
Song BY, et al. Pharmacopuncturology 2nd ed. Seoul:Elsevier Korea. 2011:3-5.

25. Kim HJ, Ji YS, Lee SM, Jeon JH, Kim YI. A Systematic Review of Clinical Study of Bee Venom Acupuncture. The Acupuncture. 2013; 30(4):151-159. 